\title{
ASSESSMENT OF THE ROLE OF 128 ROW MULTI DETECTOR COMPUTED TOMOGRAPHY IN THE EVALUATION OF CORONARY ARTERY BYPASS GRAFTS PATENCY
}

\author{
Khaled Mohamed El Gerby*, Mohamed Ibrahim Amin* Mohamed Ibrahim Amin Mousa** \\ Radiology* \& cardiology**, Departments, Faculty of Medicine, Zagazig \\ University, Egypt.
}

\begin{abstract}
Background: The recently developed 128 row multidetector CT had made a revolution in coronary artery bypass grafts
(CABG) patency evaluation due to high temporal and spatial resolution beside it is being non invasive.

Subjects and Methods: 50 patients were enrolled for ECG gated CT of coronary arteries bypass grafts between January 2015 and May 2017.

Patients underwent conventional coronary angiography within 2 weeks of MDCT exam. Administration of beta blocker and nitroglycerin was done prior to CT exam. Breath hold training was mandatory.

Results: 46 grafts were arterial and 74 were venous. 36 of the arterial grafts (78.2\%) were patent, $6(13 \%)$ were significantly narrowed and $4(8.8 \%)$ were completely occluded. Regarding venous grafts, $52(70.2 \%)$ of them were patent, $14(19 \%)$ were significantly narrowed and $8(10.8 \%)$ were completely occluded. CT angiography compared to the conventional angiography as a gold standard technique gave us a sensitivity of $100 \%$, a specificity of about $98 \%$ and an accuracy of about $93.6 \%$ in the assessment of any type of coronary artery grafts.

Conclusion: MDCT is considered the imaging modality of choice at the current days to evaluate the patency and stenosis of coronary artery bypass grafts than the conventional coronary angiography due to its multiple drawbacks.

Keywords: MDCT, global left ventricular function, Coronary Artery Disease

Corresponding author:

Mohamed Ibrahim Amin. Tel: 01145465001. Email: aasser2012@ gmail.com.
\end{abstract}

\section{INTRODUCTION}

$\mathbf{T}$ he conventional coronary angiography considered the reference standard to assess the coronary artery stenosis, stent stenosis and the patency of coronary artery bypass grafts. The adverse effects of such technique made the noninvasive alternatives, such as CT and MRI, more readily usable at such examination; especially they yielded good diagnostic accuracy in coronary imaging [1].

With the availability of multidetector CT scanners, cardiac imaging is becoming a practical application, possibly due to the increased gantry rotation speed, enhanced detector multiplicity and improved ability to recombine data from various phases [2].

Role of cardiac CT imaging includes detection of arterial calcifications on nonenhanced scans and demonstration of vascular stenosis on coronary CT angiograms. Technical factors such as a slow heart rate, a short scanning time, sub millimeter spatial resolution, high temporal resolution and reconstruction of multiple image data sets at various intervals in the cardiac cycle result in optimal visualization of the coronary arteries. In cases with increased heart rate, the most convenient image quality accomplished at the end-systolic and early-diastolic intervals; in cases with low heart rate, the best images are achieved with mid-diastolic intervals [3].

\subsection{Patients}

SUBJECTS AND METHODS

A total of fifty patients were included in the study from January 2015 to August 2017. They were 48 males (96\%) and 2 females (4\%), their age ranged from 41 to 71 years. The protocol for the CT exam and an informed consent form used in this study were approved by the Institutional Review Board (IRB) of Zagazig University. Patients signed a written informed consent and filled a written survey including demographic and clinical data. Patients were referred from the cardiology outpatient clinics \& Cardio-thoracic Hospital inpatient, Zagazig University hospitals. The inclusion criteria included patients with post operative need to assess the patency of their grafts, rule out any post operative complications, assess the progression of the disease in the native arteries and those who need further surgical intervention. Patients with renal insufficiency or presented with arrhythmia were excluded. 
All patients were subjected to the following:

A) Full clinical history including personal history (age, sex, occupation, residency and special habits as smoking) and past history (history of CABG operation).

B) Patients underwent conventional coronary angiography whenever indicated for graft patency assessment based on clinical state or suspicion of occlusion.

C) Within the following 7-10 days, patients underwent MDCT coronary angiography, using Philips Ingenuity core 128 TM (v3.5.7.25001, Philips healthcare systems, Netherlands) at radiology department, Zagazig University Hospital.

\subsection{Methods of MDCT exam}

\section{Patient preparation}

Patients were asked to fast 4-6 hours prior to the examination.

\section{Beta Blockers}

To reduce the heart rate (below $65 \mathrm{bpm}$ ), oral administration of a beta blocker 1 hour before CT scanning (100 mg atenolol) was applied. The onset of action of is approximately 30 min.

\section{Breath-hold Training}

To improve the temporal resolution, patients were practiced to take deep breath then hold it for about 20 seconds in order to reduce the heart rate, as well as during the actual scan to reduce respiration motion artifact.

Positioning and ECG

The patient is asked to lie supine on the CT table with the arms above the head. The electrodes of the ECG leads are put near to the heart but not included within the scan field (to avoid artifacts). Three electrodes are put as follows: One along the right clavicle represents the right arm, the second one along the left clavicle represents the left arm and the last one inferior to nipple represents the lower limb.

Contrast Agent

Through $18 \mathrm{G}$ canula into the antecubital vein of the right upper limb the intra venous contrast was injected according to the patient weight we adjusted the contrast agent injection rate (usually $5-6 \mathrm{ml} / \mathrm{sec}$ ) \& the required amount of contrast $(1-1.5 \mathrm{ml} / \mathrm{kg}$, mean about $100 \mathrm{ml}$ for an $80 \mathrm{~kg}$ body Khaled M.; et al.... weight). First we inject contrast agent, then saline chaser $(50 \mathrm{ml}$ by the same flow rate of contrast injection) simultaneously, using a dual-syringe injector.

Nitroglycerin

Sublingual nitroglycerin administration was routine at all exams. The onset of action is about 10-20 s after administration, and its effect lasts for about 10-30 min.

Planning the Scan

The scan range applied was started approximately just above the level of the clavicle to include the full length of any arterial grafts (left or right internal mammary artery grafts) down to the diaphragmatic copula to secure the vicinity of the inferior \& posterior heart surface.

Starting the Scan

We used the following parameters: Detector collimation $16 \times 0.75 \mathrm{~mm}$, tube voltage 120 $140 \mathrm{kV}$ and tube current $600-800 \mathrm{~mA}$, based upon the body mass index. Pitch of 0.2-0.3, rotation time 0.42 seconds, reconstruction slice width $0.6 \mathrm{~mm}$ and increment $0.5 \mathrm{~mm}$.

We applied the bolus tracking technique. Simply it monitors contrast arrival and trigger the scan so long the pre-determined threshold achieved (100 HU). We put the ROI (region of interest) at the descending thoracic aorta just below the level of tracheal bifurcation. There is delay time of 6 seconds between the contrast arrival to the determined threshold and triggering the scan. We used a prospective ECG gated coronary scan.

Image processing \& reconstruction

Images were reconstructed at $0,30,35,75 \&$ 80 of the R-R interval of the cardiac cycle. The images were then post-processed using the Philips Extended intellispace ${ }^{\mathrm{TM}}$ portal Workstation. Multiple reformations multiplanar reformation (MPR), curved planar reformation (CPR), maximum intensity projection (MIP), shaded-surface display (SSD) and volume rendering (VR) to delineate the grafts, native coronary arteries, cardiac and extra cardiac changes.

\subsection{Statistical Analysis}

The findings of CT imaging studies were evaluated and correlated to the conventional coronary angiography. Sensitivity, specificity and diagnostic accuracy were calculated. All 
data manipulation and analysis were performed using the 17th version of SPSS (Statistical Package for Social Sciences).

\section{RESULTS}

This study included 50 patients with prior CABG surgery.

The mean age of included patients was 56 with an age range between 41 and 71 years. Male patients were $48(96 \%)$ while females were $2(4 \%) .40(80 \%)$ had diabetes mellitus, $42(84 \%)$ had hypertension, $45(90 \%)$ were smokers, $35(70 \%)$ had dyslipidemia.

A total of 120 CABGs were imaged at this study and further analyzed for patency. Within the grafts examined 46 were arterial and 74 were venous. Out of the arterial grafts, $36(78.2 \%)$ were patent, $6(13 \%)$ were significantly narrowed and $4(8.8 \%)$ were completely occluded. Regarding the venous grafts, $52(70.2 \%)$ of them were patent, 14 $(19 \%)$ were significantly narrowed and 8 $(10.8 \%)$ were completely occluded.

Arterial Grafts- Arterial grafts may be insitu e.g. left internal mammary artery (LIMA) \& right internal mammary artery (RIMA) or free e.g. radial artery. This study included 39 $(84.7 \%)$ insitu grafts and $7(15.3 \%)$ free grafts.

Regarding the insitu arterial grafts, 33 of them $(84.6 \%)$ were LIMA grafts and 6 (15.4

\section{List of tables}

Table 1) shows demography of the cases.

\begin{tabular}{ll}
\hline Factor & Number \\
\hline Age & $41-71$ years old with the mean: $56 \mathrm{y}$ \\
\hline Sex & Male: $48(96 \%)$ \\
& Female: $2(4 \%)$ \\
\hline Diabetes Mellitus & $40(80 \%)$ \\
\hline Hypertension & $42(84 \%)$ \\
\hline Smoking & $45(90 \%)$ \\
\hline Dyslipidemia & $35(70 \%)$ \\
\hline
\end{tabular}

$\%)$ were RIMA, 34 of them (87.5\%) were connected to the left anterior descending (LAD), $2(5 \%)$ to the obtuse marginal (OM), $2(5 \%)$ to RCA, $1(2.5 \%)$ to the $1^{\text {st }}$ diagonal branch of LAD. $32(82 \%)$ were patent, 4 $(10.2 \%)$ were significantly narrowed and 3 (7.8\%) were completely occluded.

For the LIMA grafts which were the commonest type, $30(93.7 \%)$ of them were connected to the LAD. $28(84.8 \%)$ of LIMA grafts were patent, $3(9 \%)$ were significantly narrow and $2(6.2 \%)$ were occluded.

Four of the free grafts $(57 \%)$ were seen patent, $2(28.5 \%)$ were narrow and $1(14.5 \%)$ was occluded.

Venous Grafts- 74 venous grafts were included at this study. 20 of them (27\%) were to the LAD, $35(47 \%)$ to the OM, $10(14 \%)$ to RCA, $5(6.7 \%)$ to the first diagonal and 4 $(5.3 \%)$ to the PDA. $8(10.8 \%)$ were occluded grafts whose their landing site could not be identified.

Conventional angiography is considered the gold standard technique. The sensitivity of the MDCT when compared with conventionalangiography at this study was $100 \%$, while the specificity about $96 \%$ and the diagnostic accuracy about $93.6 \%$. 
(Table 2) represents types and numbers of the arterial grafts included in the study and their degree of patency.

\begin{tabular}{|c|c|c|}
\hline \multicolumn{3}{|c|}{ Arterial grafts (46) } \\
\hline Free: $7(15.3 \%)$ & & In situ: $39(84.7 \%)$ \\
\hline $\begin{array}{l}\text { Degree of patency } \\
=4(57 \%) \text { patent } \\
=2(28.7 \%) \text { narrow } \\
=1(14.3 \%) \text { occluded }\end{array}$ & $\begin{array}{l}\text { Types: } \\
=\text { LIMA: } 33(84.6 \%) \\
=\text { RIMA: } 6(15.4 \%) \\
\text { Anastomosis: } \\
=34(87.5 \%) \text { to LAD } \\
=2(5 \%) \text { to OM } \\
=2(5 \%) \text { to RCA } \\
=1(2.5 \%) \text { to D1 } \\
\text { Degree of patency } \\
=32(82 \%) \text { patent } \\
=4(10.2 \%) \text { narrow } \\
=3(7.8 \%) \text { occluded }\end{array}$ & \\
\hline
\end{tabular}

Degree of patency of all arterial grafts:

$=36(78.2 \%)$ patent

$=6(13 \%)$ narrow

$=4(8.8 \%)$ occluded

(Table 3) represents types and numbers of the venous grafts included in the study considering their degree of patency.

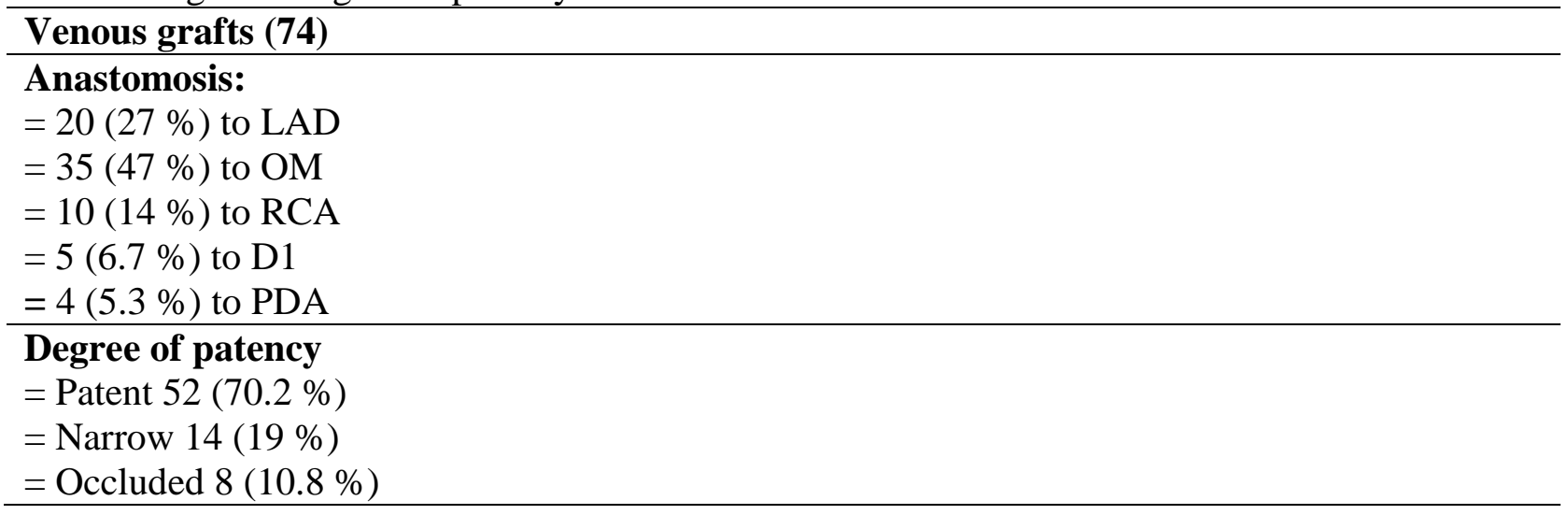

(Table 4) shows sensitivity, specificity \& diagnostic accuracy of the MDCT coronary angiography in evaluation of coronary artery bypass grafts patency.

\begin{tabular}{l|l}
\hline & \\
\hline Sensitivity & $100 \%$ \\
\hline Specificity & $98 \%$ \\
\hline Accuracy & $93.6 \%$ \\
\hline
\end{tabular}

Figures legend 


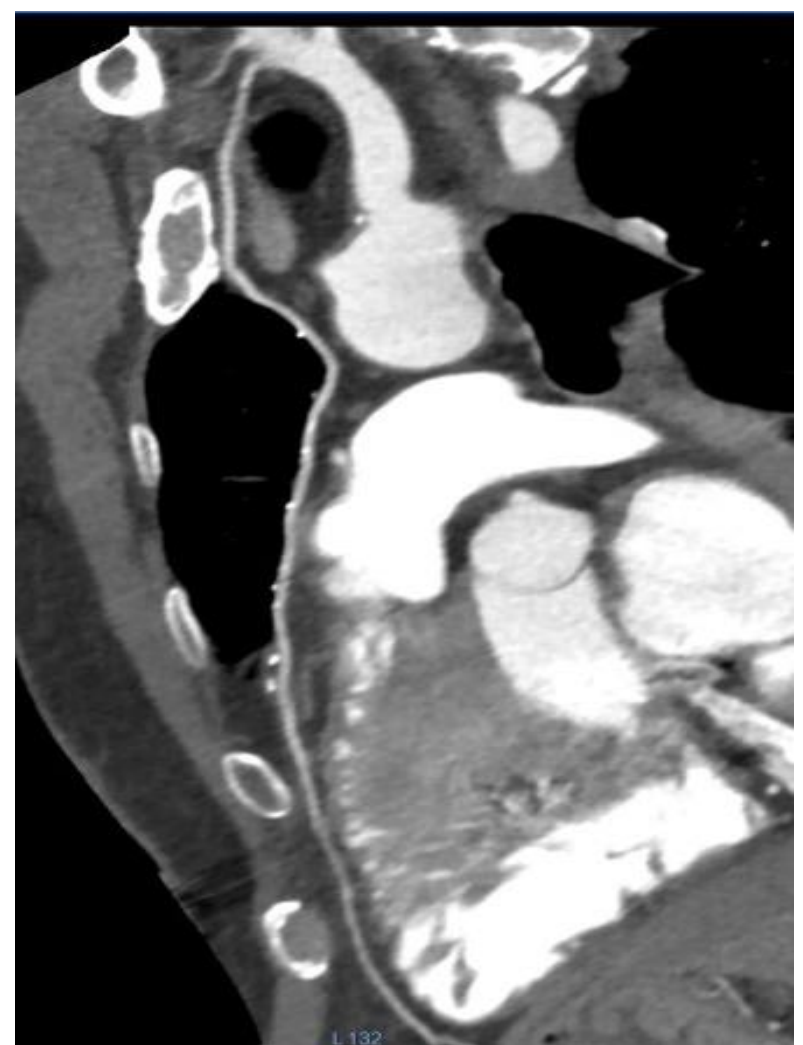

Fig. 150 years old male presented with follow up for LIMA (left internal mammary artery) to LAD (Left anterior descending) graft. Curved planar reformatted maximum intensity (MIP) images shows good contrast opacification along the LIMA to LAD graft with distal opacification of the Lad down to the cardiac apex.

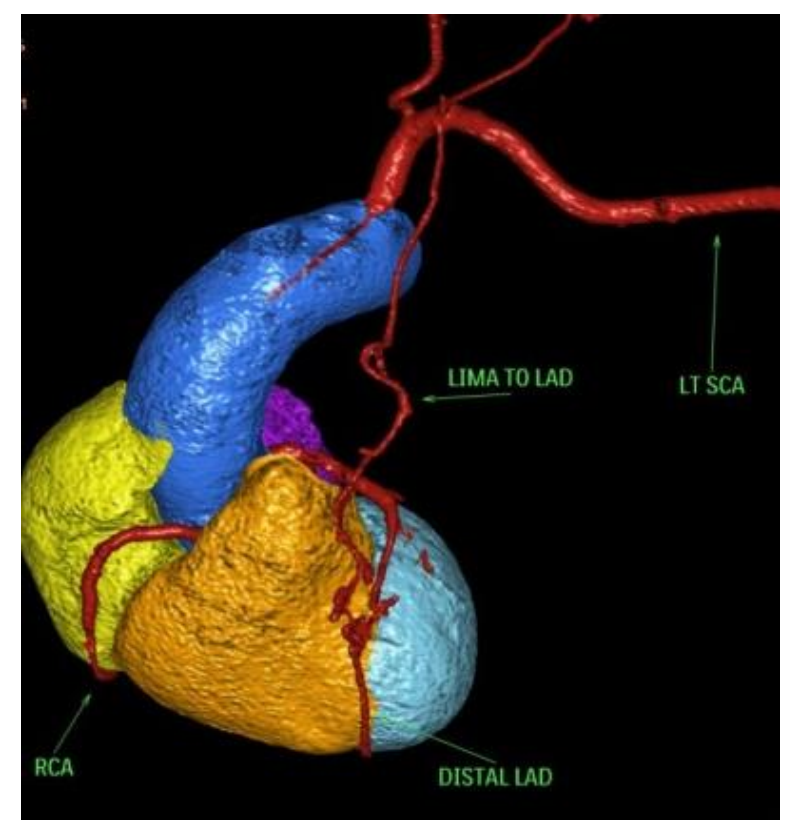

Fig. 2 The same case with color coded VR image shows the patency of the origin (from the left subclavian artery), body \& distal anastomosis of the LIMA to LAD graft. 


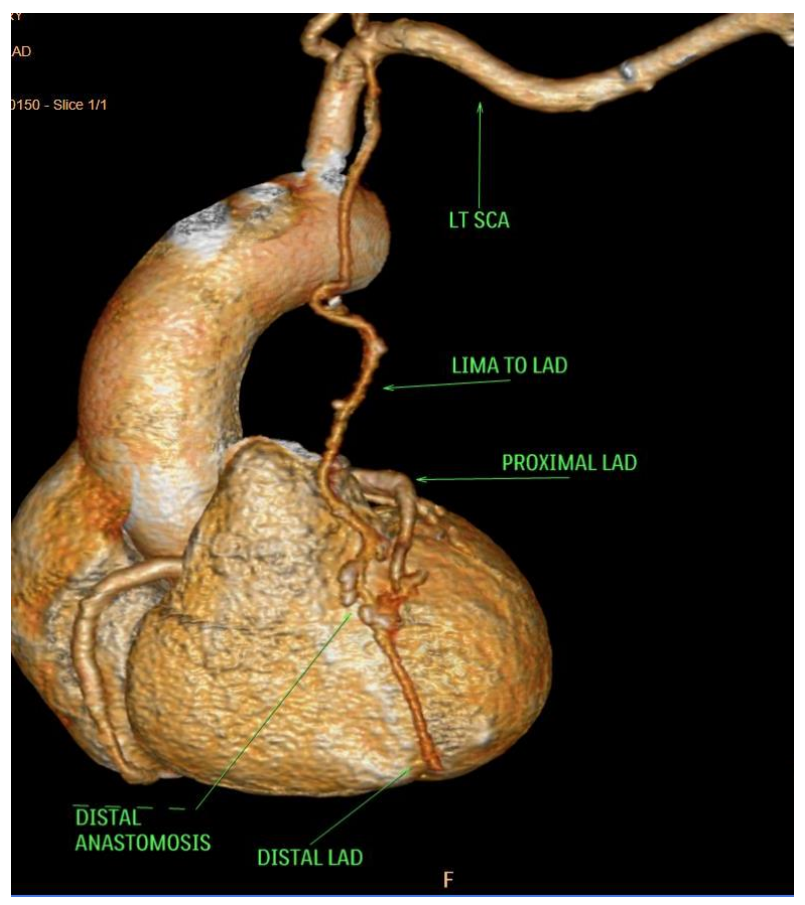

Fig. 3 The same case with 3D VR image shows the patency of the origin (from the left subclavian artery), body \& distal anastomosis of the LIMA to LAD graft.

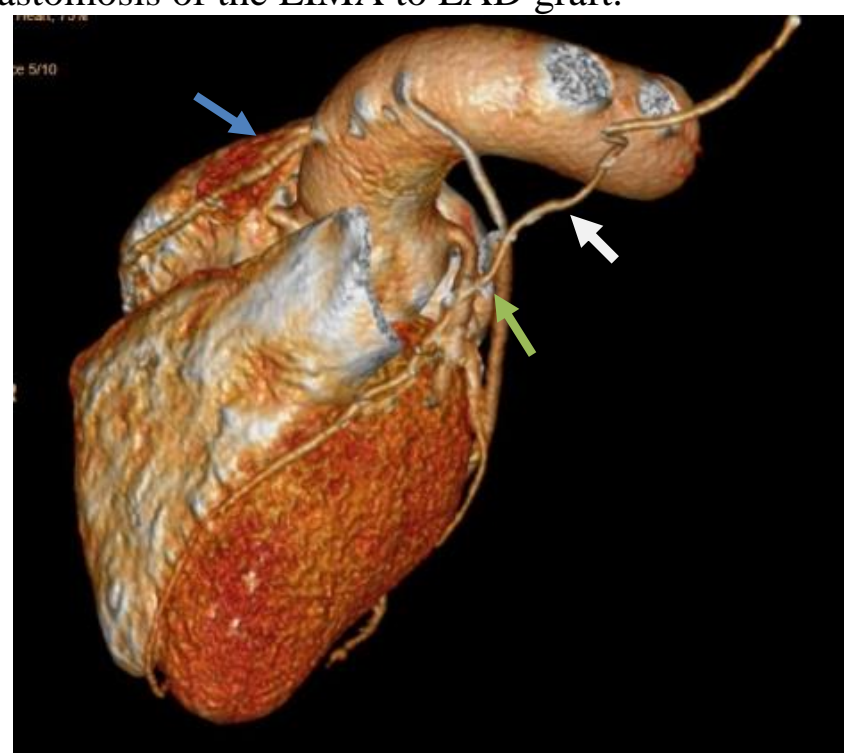

Fig. 455 years old heavy smoker male presented for routine follow up of 3 coronary artery by pass grafts. 3D VR image shows intact origins, bodies \& distal anastomosis sites for the LIMA to LAD (white arrow), SVG (saphenous venous graft) to OM (Obtuse marginal branch of left circumflex artery) (green arrow) \&SVG to RCA (blue arrow). 


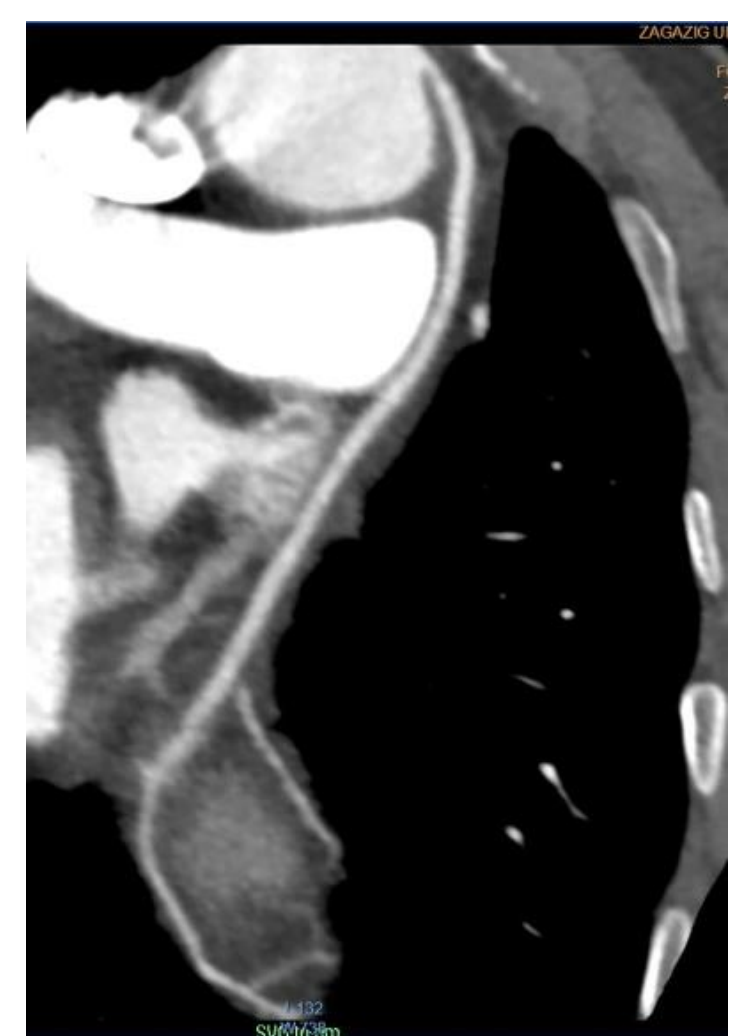

Fig. 5 Curved planar reformatted maximum intensity projection (MIP) image for the same case shows good contrast opacification along the SVG to OM graft and good opacification of the distal LCX (left circumflex artery).

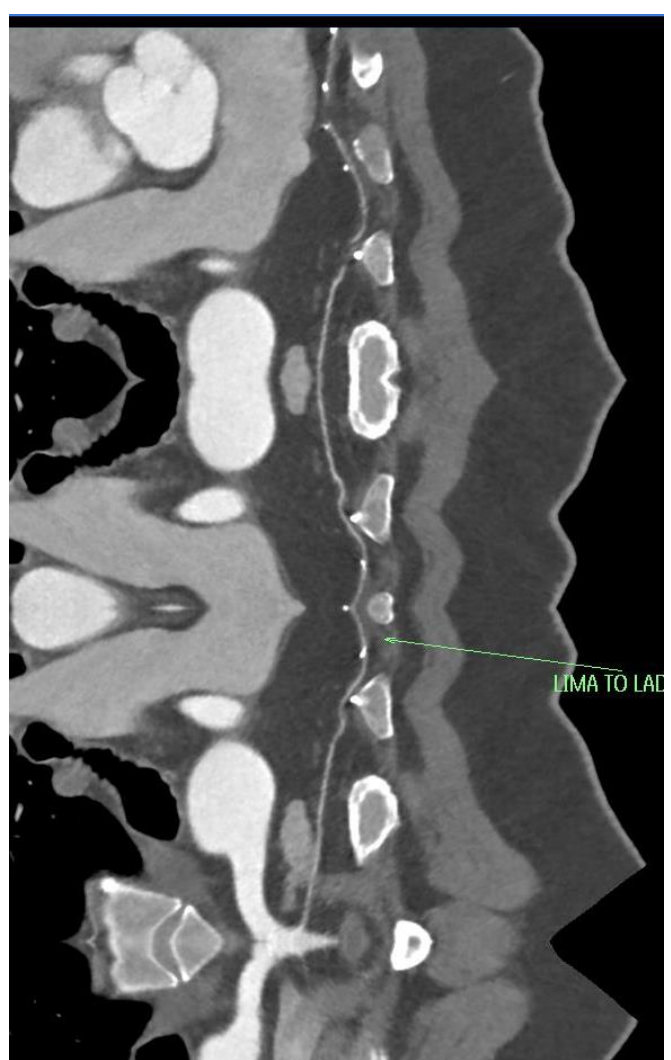

Fig. 6 Curved planar reformatted maximum intensity projection (MIP) image for the same case shows good contrast opacification along the LIMA to LAD graft and good opacification of the distal LAD (left anterior descending artery). 


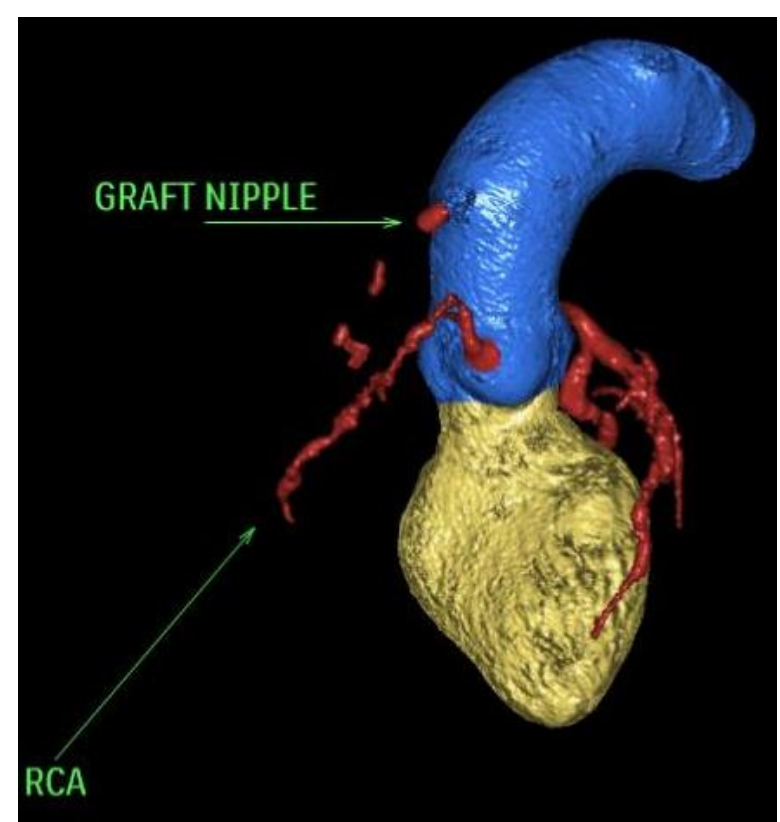

Fig. 766 years old male with SVG to RCA graft 12 years age, presented with agonising chest pain). Color coded VR image shows contrast opacification along the origin of the graft at the ascending aorta, interrupted course \& non opacified distal anastomosis denoting graft failure/occlusion. Non opacified mid/distal RCA (right coronary artery).

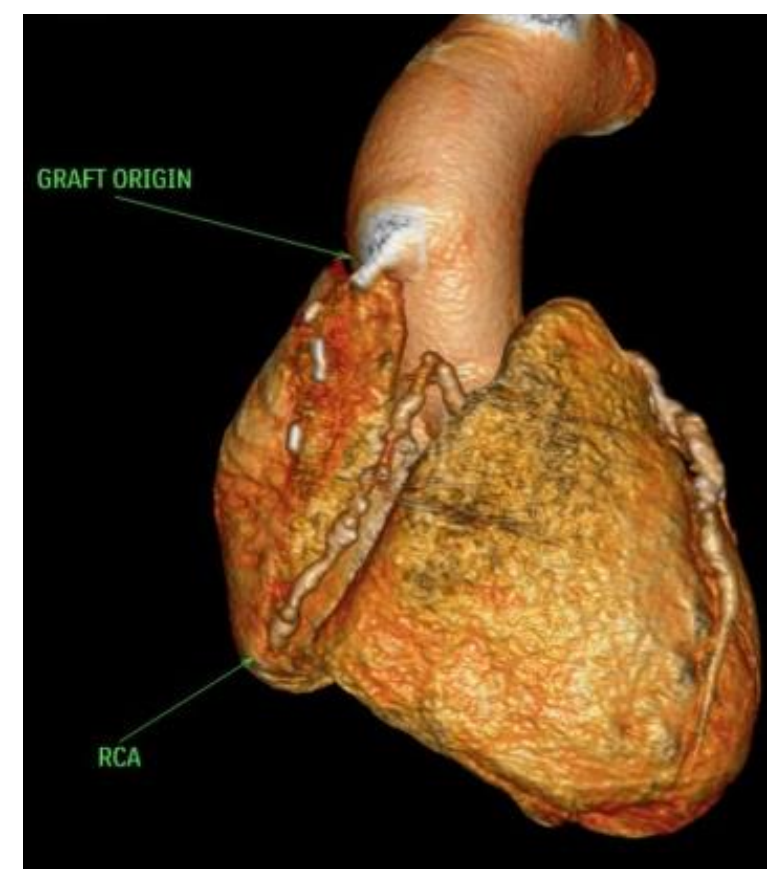

Fig. 8 3D VR image for the same case shows the origin of the graft at the ascending aorta, interrupted course, metallic clips \& graft failure at the distal anastomosis (RCA: right coronary artery).

\section{DISCUSSION}

Nowadays, the coronary artery bypass graft surgery (using either venous or arterial grafts), is considered an effective method of treatment for those with multi-vessel coronary artery lesions. There are multiple variables that can affect the successful outcome of such surgery; the most important of them is the 
graft short- and long-term patency rates. High patency rates and good long terms outcomes, depends upon the graft quality [4].

Although the reference standard to evaluate the patency of such grafts is the conventional angiography, it poses multiple disadvantages, the most important are invasiveness and patient discomfort. From that point the need to use a less invasive imaging modality has become a must for such purpose [5].

Those who underwent that kind of surgery are usually facing a big deal in the diagnostic issues for the angiographer. Calcifications, tortuosity, diffusely affected coronary arteries and degenerated grafts add more difficulty in accurate lesions delineation. The evaluation at such cases in a noninvasive manner unfortunately is more difficult, due to some limitations as well [6].

Multi-detector row CT currently is becoming the best choice to noninvasively image the heart, coronary arteries and their grafts and of course, multiple studies confirmed that role.

Great development of the 128- row CT scanner when compared with older generation scanners include higher spatial resolution (due to sub-millimeter collimation), higher temporal resolution (due to shorter gantry rotation time), plus significant scan time lessening. Despite the recent technical advances, the 128-detector row CT coronary angiography is still sensitive to arrhythmia. Persistent irregular cardiac rhythm such as atrial fibrillation and frequent extra-systoles preclude MDCT coronary angiography. However, motion artifacts owing to mild arrhythmia (e.g. single ventricular extrasystole) can be diminished by manual repositioning the reconstruction windows [7].

Regarding the venous grafts, and according to Frazier et al 2005 [8] the post operative occlusion rate along the $1^{\text {st }}$ year is $12-20 \%$.After 5 years post operative, the rate decreases to $4 \%$ every year. This rate for the IMA grafts becomes $10 \%$ after 10 years post operative according to Loopin 1996 [9].

According to De Feyter et al, 2008 [10], up to $10 \%$ of grafts become occluded during the perioperative period. After 10 Khaled M.; et al.... years, occlusion rates of $59 \%$ and $17 \%$ for venous and arterial grafts respectively. About $40 \%$ of patients who undergo bypass surgery will experience recurrence of symptoms within 6 years and $25 \%$ of bypass grafts are found to be occluded at 5 years follow up.

Venous grafts are still the most widely used type of grafts due to their abundance. In our study also, venous grafts were the most common type of grafts. It represented 74 of the 120 grafts, nearly about $61.6 \%$. Yet, there is high rate of complications with venous grafts due to their high rate of occlusion and narrowing. Our study showed that $19 \%$ of the venous grafts were significantly narrowed and about $10.8 \%$ of them were totally occluded. The OM branch was the commonest site for venous graft landing (47\%) followed by the LAD $(27 \%)$.

The insitu arterial grafts are used more common than the free arterial grafts, as the later has higher rate of complications. In our study, $12.5 \%$ of the arterial grafts were free arterial grafts. There was significant narrowing in $14 \%$ of the free arterial grafts, and a $14 \%$ rate of occlusion, raising the rate of complications in free arterial grafts to about $28 \%$ in our study.

As for the insitu arterial grafts, they are one of the most widely used grafts (84.7 $\%$ of the arterial grafts in our study), especially at the relatively younger patient due to their high patency rates after a long time, yet they are not abundant. The LIMA is the most widely used insitu arterial graft (84.6 $\%$ of the insitu arterial grafts in our study). Due to its arterial nature, it has less incidence rate of complications. The LIMA grafts showed an $84.8 \%$ rate of patency in our study compared to $90 \%$ rate of patency according to Flohr 2005 [11]. There was a $9 \%$ rate of significant narrowing and a $6.2 \%$ rate of occlusion in our study.

The LAD is the most common site for landing of the LIMA graft, as it is the most important coronary artery branch supplying the left ventricle and due to other anatomical considerations as they are both anteriorly located in the chest. $93.7 \%$ of our LIMA grafts ended at the LAD. 
The mean time after the CABG surgery was about 4 years, so we were not able to assess any of the early complication such as acute and subacute graft occlusion, sternal infection and operative bed collections. All these complications may have been detected if the MDCT angiography was done right after surgery. We had been dealing with chronic complications as we were dealing with outpatient based clinics.

In a prospective study done by Romagnoli $A$ et al 2010 [12], they assessed 78 patients with a total of 213 bypass grafts using ECG-gatedMDCT coronary angiography (64 row), they compared the results with conventional coronary angiography as the standard of reference. According to their findings multi-detector rowCT angiography presented a good diagnostic performance, regarding the assessment of occlusion of the grafts (sensitivity: $100 \quad \% \quad \&$ specificity: 97\%), findings that were close to our study (sensitivity: 100\% \& specificity: 98\%).

In a study done by Jürgen et al 2004 [13], they used thick sections, so get shorter time that is suitable for the patients to hold their breath after inspiration. They had also determined the optimal reconstruction interval. However, as a trade off, they got a reduced quality of the images. This was aggravated by artifacts of the operative metallic clipsin five of $62(8 \%)$ of the patients. They attributed the low frequency of such artifact to the on use of differentsurgical techniques at such study.

The quality of images for different parts of the coronary artery bypasses grafts mostly not uniform. The bodies and the distal anastomosis of the grafts are more prone to heart beat-related artifacts while the proximal segments are hardly affected [14].

The radiation dose is the greatest disadvantage of the retrospective ECG-gated MDCT coronary angiography. The acquisition of data is acquired using continuous X-ray exposure with overlapping helical pitch, yielding a higher dose than thatof the prospective ECG-gated exam.The mean effective dose value for MDCT coronary angiography was 5 times greater than the value for conventional coronary angiography. A dose reduction by up to $48 \%$ is possible according to Jakobs et al 2002 [15], through reduction of theoutput of the CT scanner $\mathrm{X}$ ray tube through the heart phases that are unlikely to be targeted by the ECGgatedreconstruction (except 50\%-70\%).

To get verified data acquisition in this study, we did not make changes to the tube outputat different phases of the cardiac cycle.

Hamon et al [16], had made a study in 2008, they performed a meta-analysis to assess the accuracy of 16- and 64-row detectors coronary angiography in cases of coronary artery bypass grafts. The results of their metaanalysis demonstrate that obstructive bypass graft disease can be detected by using at least a 16-section CT with a sensitivity between 80 and $96 \%$ for significant stenosis (defined as a diameter reduction of $>50 \%$ ) and specificity between 85 and 95\% [17].

This performance is better than for non graft coronary arteries as shown in a recent metaanalysis by Hamon et al in 2006 [18], in which the sensitivity was $82 \%$ and specificity was $91 \%$. The results of our study showed sensitivity $100 \%$ and specificity $98 \%$ that were almost the same was a study done using 64 row MDCT done by Monem SA et al., in 2012 [19], with sensitivity $100 \%$ and specificity $96.4 \%$.

There are multiple differences that can help to explain the improved performance between bypass grafts and native coronary arteries. The larger diameter of the graft conduits, make them to calcify less frequently. Beside they are relatively immobile, feature that make their imaging is easier. However, they have higher rate of occlusion than stenosis. The diagnosis of obstruction is considered to be clearer cut at such cases.

Compared with conventional angiography, CT angiography is less costly, faster, does not require assembly of an angiographic team to perform the study and is generallymore available 24 hours a day. It permits a wider variety of manipulations of the volumetric data set for image display and analysis in contrast to the limited projections routinely obtained during conventional 
angiography, and has fewer potential complications [20].

MDCT is considered a less invasive technique, and this is considered a valuable advantage over coronary angiography. In cases of CABG conventional coronary angiography considered more difficult than for native vessels, being longer in duration and consuming more amount of contrast agent, so there is an increase in the complication rate [21].

In many cases it is hard to trace the grafts origin to selectively explore them. Nonetheless, MDCT determines an accurate position of the existing grafts and any aortic intimal calcifications. Those requiring advanced aortic valve surgery, CT coronary angiography is more helpful to assess the aortic root (including size and calcification) rather than the conventional angiography [22].

Though MDCT coronary angiography exhibits many advantages, yet it poses multiple limitations. The worst of them is its inability to fully assess a number of scanned grafts. Reasons for non assessability include cardiac motion, respiratory artifacts, poor opacification and the presence of surgical clips. However, 128-row detectors CT scanner, yields an increase in the temporal, spatial resolutions and shorter acquisition time that help to improve the rate of fully assessable scanned grafts. With a four-row CT scanner, 40 seconds are needed for a complete assessment of a graft. With a 16row CT scanner, less than 30 seconds is needed, and less than 16 seconds for 128-row CT scanner. The existence of surgical metal clips and thorough evaluation of the grafts' distalanastomoses remain a challenging issue at all CT scanners generations.

In order to follow-up a case with CABG, assessment of the bypass grafts and also the native coronary arteries is mandatory. Although the diagnostic accuracy is reasonable at such cases, but there many factors that make the accomplishment of a full and complete assessment difficult, these include the calcium deposits in the vascular walls and motion artifacts, especially those with increased heart rates. Patients that have Khaled M.; et al.... native vessels with earlier stage of atherosclerotic disease are more easily assessed than those patients who have undergone bypass surgery. With more advancement in the atherosclerotic degenerative process, small, diffusely narrow vessel with abundant wall calcifications, adds more difficulty to properly assess the vessel lumen [23].

The general limitations of the cardiac MDCT include the following: The use of potentially nephrotoxic agents, radiation exposure and unavoidable exclusion of cases with atrial fibrillation or severe respiratory disease. The specific limitations include: defective data about the graft flow characteristics and function. As well as, the surgical clips, severe calcifications and extensive atherosclerotic disease, all of which hinder the full assessment of the native vessels and to a small degree their grafts. Notably as well, the specific limitations of such examination include an increase of the acquisition volume that leads to an increase in the radiation exposure. Increased amount of contrast media raises the nephrotoxicity risk.

Current advances using 256 and 320 detector row as well as dual source CT machines can open frontiers in the assessment of the heart, coronary arteries and coronary grafts. Faster examinations with much less radiation dose are now possible.

\section{CONCLUSION}

Although the standard of reference to evaluate the patency of coronary artery bypass grafts is the conventional coronary angiography, the existence of its multiple drawbacks had made the less invasive MDCT the imaging modality of choice at the current days especially when comparing as well the effective radiation dose burden. Although there are limitations for the CT technique, the current advances at the newer versions of CT scanner can yield more better results that are highly comparable with conventional angiography that presents a good imaging substitution modality with almost the same diagnostic accuracy.

\section{REFERENCES}

1. Kim WY, Danias PG, Stuber M, et al. Coronary magnetic resonance angiography for 
the detection of coronary stenosis. NEngl J Med; 2001: 345:1863-1869.

2. Choi B, Yoo $\mathbf{K} \&$ Choi $\mathbf{D}$ et al. The comparison of the graft patency after coronary artery bypass grafting using coronary angiography and multi-slice computed tomography. Eur J CardiothoracSurg 2003: 24:86-91.

3. Achenbach S, Ropers D, \& Kuettner A et al. Contrast-enhanced coronary artery visualization by dual-source computed tomography - initial experience. Eur J Radiol 2006: 57: 331-335.

4. Mahnken AH, Seyfarth T \& Flohr T et al. Flat-panel detector computed tomography for the assessment of coronary artery stents: phantom study in comparison with 16-slice spiral computed tomography. Invest Radiol 2005: 40(1):8-13.

5. Mahesh M. Cardiac imaging-technical advances in MDCT compared with conventional $\mathrm{x}$-ray angiography. US Cardiol 2006: 115-118.

6. Marc Dewey. Coronary CT Angiography 1st edition Springer-Verlag Berlin Heidelberg 2009: p 119.

7. Cademartiri F, Maffei E \& Palumbo AA et al. Influence of intra-coronary enhancement on diagnostic accuracy with 64-slice CT coronary angiography. EurRadiol; 2008: 18:576-83.

8. Frazier A, Fauzia Q \& Katrina MR et al. Coronary Artery Bypass Grafts: Assessment with Multidetector CT in the Early and Late Postoperative Settings. RadioGraphics; 2005: 25:881-896.

9. Loop FD. Internal thoracic artery graft: biologically better coronary arteries. N Engl J Med; 1996: 334: 263-265.

10. De Feyter, Francesca $\mathbf{P} \&$ Nico $\mathbf{R M}$ et al. Diagnostic Performance of Coronary CT Angiography by Using Different Generations of Multisection Scanners: Single-Center Experience. Radiology: 2008: Volume 246: Number 2-February.

11. Flohr TG, Schaller S \& Stierstorfer BM et al. Multi-detector row CT systems and imagereconstruction techniques. Radiology; 2005: 235:756-773.

12. Romagnoli A, Patrei A \& Mancini A et al. Diagnostic accuracy of 64-slice CT in evaluating coronary artery bypass grafts and of the native coronary arteries. Radiol med 2010: 115:1167-1178.

13. Jürgen KW, Dominik W, Richard $K$ et al. Coronary Artery Bypass Grafts: ECG-gated Multi-Detector Row CT AngiographyInfluence of Image Reconstruction Interval on Graft Visibility. Radiology; 2004: 232:568-577.

14. Kopp AF, Schroeder S \& Kuettner A. et al. Non invasive coronary angiography with high resolution multi-detector row computed tomography. Eur Heart J, 2002: 23; 1714.

15. Jakobs TF, Becker CR \& Ohnesorge B et al. Multislice helical CT of the heart with retrospective ECG gating: reduction of radiation exposure by ECG-controlled tube current modulation. EurRadiol; 2002: 12:1081-1086.

16. Hamon M, Biondi-Zoccai G \& Malagutti $P$ et al. Diagnostic performance of multislice spiral computed tomography of coronary arteries as compared against conventional invasive coronary angiography: a meta-analysis. J AmCollCardiol 2006: 48:1896-1910.

17. Langerak SE, Vliegen HW \& de Roos A et al. Detection of vein graft disease using highresolution magnetic resonance angiography. Circulation; 2002: 105:328-333.

18. Hamon M, Lepage $\mathbf{O}$, Malgutti $\mathbf{P}$, et al. Diagnostic performance of 16 and 64 section spiral CT for coronary arterybypass graft assessment: meta-analysis. Radiology; 2008: 247:679-86.

19. Monem SA, Nasr A. MS \& Algebalay A. MW et al. Sixty four multi-detector CT coronary angiography: An easy and accurate method to detect graft patency post CABG. EJRNM; 2012: 43:377-381.

20. Asai T, Ochi M \& Yokoyama. Off-Pump Coronary Artery Bypass, Springer, 2015: Oct 6.

21. Ropers D, Baum U \& Pohle K, et al. Detection of coronary artery stenoses with thinslice multi-detector row spiral computed tomography and multiplanar reconstruction. Circulation 2003: 107:664-6.

22. Tintoiu IC, Underwood MJ \& Cook SP et al. Coronary Graft Failure: State of the Art, Springer, 2016: Mar 24.

23. Turkvatan A, Biyikoglu SF, Bujukbayraktar FG et al. Noninvasive evaluation of coronary artery bypass grafts and native coronary arteries; is 16-slice multidetector CT useful? DiagnIntervRadiol; 2009: 15:43-50. 